JOURNAL OF

SYMPLECTIC GEOMETRY

Volume 2, Number 1, 1 - 24, 2003

\title{
DISCRETE TORSION AND TWISTED ORBIFOLD COHOMOLOGY YONGBIN RUAN
}

\section{Introduction}

One of the remarkable insights of orbifold string theory is an indication of the existence of a new cohomology theory of orbifolds containing the so-called twisted sectors as the contribution of singularities. Mathematically, such an orbifold cohomology theory has been constructed by Chen-Ruan $[\mathbf{C R}]$. The author believes that there is a "stringy" geometry and topology of orbifolds whose core is orbifold cohomology. One aspect of this new geometry and topology is the twisted orbifold cohomology and its relation to discrete torsion. Again, the twisting process has its roots in physics. Physicists usually work over a global quotient $X=Y / G$ only, where $G$ is a finite group acting smoothly on $Y$. A discrete torsion is a cohomology class $\alpha \in H^{2}(G, U(1))$. Physically, a discrete torsion counts the freedom to choose a phase factor to weight the path integral over each twisted sector without destroying the consistency of string theory. For each $\alpha$, Vafa-Witten $[\mathbf{V W}]$ constructed the twisted orbifold cohomology group $H_{o r b, \alpha}^{*}(X / G, \mathbf{C})$.

Vafa-Witten suggested that discrete torsion and twisted orbifold cohomology relate to desingularization. Recall that there are two methods to remove singularities, resolution and deformation. Both play important roles in the theory of Calabi-Yau 3-folds. A smooth manifold $Y$ obtained from an orbifold $X$ via a sequence of resolutions and deformations is called a desingularization of $X$. In string theory, we also require the resolutions to be crepant resolutions. It is known that such a desingularization may not exist in dimensions higher than three. In this case, we allow our desingularization to be an orbifold. As we mentioned in [CR], physicists predicted that the ordinary orbifold cohomology group is the same as the ordinary cohomology group of its crepant resolution. 
Vafa-Witten proposed that discrete torsion is a parameter for deformation, and that the cohomology of the desingularization is the twisted orbifold cohomology of discrete torsion plus the possible contributions of exceptional loci of the small resolution. (A small resolution is a special kind of resolution whose exceptional locus is of codimension 2 or more.) However, this proposal immediately ran into trouble because the number of desingularizations is much larger than the number of discrete torsions. For example, D. Joyce [JO] constructed five different desingularizations of $T^{6} / \mathbf{Z}_{4}$, while $H^{2}\left(\mathbf{Z}_{4}, U(1)\right)=0$. To count these "missing" desingularizations seems to be a serious problem. We address this problem in the current paper. On the another hand, it is well-known that most orbifolds (even Calabi-Yau orbifolds) are not global quotients so it is necessary to develop the theory over general orbifolds. The theory in this paper addresses this problem as well.

First, we introduce the notion of inner local system $\mathbf{L}$ for an arbitrary orbifold. An inner local system is defined as an assignment of a flat (orbifold) line bundle $L_{(g)}$ to each sector $X_{(g)}$ satisfying certain compatibility conditions (see definition 2.1). Such compatibility conditions are designed in such a way that Poincaré duality and cup product in ordinary orbifold cohomology survive the process of twisting. The author believes that the inner local system is a more fundamental notion than discrete torsion. The twisted orbifold cohomology $H_{\text {orb }}^{*}(X, \mathbf{L})$ is then defined as orbifold cohomology with values in the inner local system (see definition 2.2). We will demonstrate that our inner local systems count all the examples constructed by D. Joyce. We make the following:

Conjecture. Suppose that $X$ is a Calabi-Yau Gorenstein orbifold. To every desingularization we can associate an inner local systems such that as additive groups the ordinary orbifold cohomology of the desingularization is the sum of twisted orbifold cohomology and contributions from the exceptional locus of the small resolution.

Our next goal is to determine the appropriate notion of discrete torsion for a general orbifold $X$. Let $X$ be an arbitrary almost complex orbifold. Our key observation is that we should use the orbifold fundamental group $\pi_{1}^{o r b}(X)$ (see definition 2.1) to replace $G$. Then discrete torsion of $X$ is defined as a cohomology class $\alpha \in H^{2}\left(\pi_{1}^{\text {orb }}(X), U(1)\right)$. Note that if $X=Y / G$ is a global quotient, then there is a short exact 
sequence

$$
1 \rightarrow \pi_{1}(Y) \rightarrow \pi_{1}^{\text {orb }}(X) \rightarrow G \rightarrow 1 .
$$

It induces a homomorphism $H^{2}(G, U(1)) \rightarrow H^{2}\left(\pi_{1}^{\text {orb }}(X), U(1)\right)$. Hence a discrete torsion in the sense of Vafa-Witten induces a discrete torsion as in this paper. The link between discrete torsion and twisted orbifold cohomology is the theorem that discrete torsion induces an inner local system and hence defines twisted orbifold cohomology. However, we emphasize that not every inner local system comes from discrete torsion (see example 5.3).

We will introduce inner local systems and the twisted orbifold cohomology ring in section 2 . Section 3 is devoted to discrete torsion. The relation between discrete torsion and the inner local system is discussed in section 4. Finally, some examples are computed in section 5. This paper can be viewed as a sequel to $[\mathbf{C R}]$. Since many constructions are similar we follow the notations of $[\mathbf{C R}]$ and only sketch the details.

This paper was completed while author was visiting Caltech. He would like to thank R. Pandharipande and the Caltech mathematics department for their financial support and hospitality. The author would like to thank E. Zaslow for bringing his attention to Vafa-Witten's paper and E. Witten for explaining to him $[\mathbf{V W}]$. He would like also thank A. Adem for many valuable discussions about group cohomology and J. Robbin and A. Greenspoon for editorial assistance.

\section{A review of ordinary orbifold cohomology}

2.1. Twisted sectors. Suppose that $X$ is an orbifold. (See $[\mathbf{C R}]$ for the formal definition.) Roughly speaking, $X$ is a topological space with a system of orbifold charts (also called uniformizing systems). Every point $p \in X$ has a system of orbifold charts of the form $U_{p} / G_{p}$, where $U_{p}$ is a smooth manifold and $G_{p}$ is a finite group acting on $U_{p}$ fixing the preimage of $p$. The group $G_{p}$ is called the local group. Note that the action of $G_{p}$ does not have to be effective. If it is (for each $p$ ), the orbifold is called effective. We denote the chart by $\left(U_{p}, G_{p}\right)$. Define the set

$$
\widetilde{\Sigma_{1} X}=\left\{\left(x,(g)_{G_{x}}\right): x \in X, g \in G_{x}\right\} .
$$

Here $(g)_{G_{x}}$ denotes the conjugacy class of $g$ in the local group $G_{x}$. The set $\widetilde{\Sigma_{1} X}$ has a natural orbifold structure where the orbifold charts are pairs $\left(X_{g}, C(g)\right)$, where $X_{g}$ is the fixed point of $g \in G_{x}$ and $C(g)$ is the centralizer of $g$. The orbifold $\widetilde{\Sigma_{1} X}$ is called the inertial orbifold of $X$ 
and plays a fundamental role in orbifold cohomology. The connected components of the inertial orbifold are called sectors.

It is instructive to write down the connected components of $\widetilde{\Sigma_{1} X}$ explicitly. By definition, the orbifold charts on $X$ satisfy the following patching condition: If $q \in U_{p} / G_{p} \cap U_{r} / G_{r}$, there is an orbifold chart $U_{q} / G_{q} \subset U_{p} / G_{p} \cap U_{r} / G_{r}$ such that the inclusion map $i: U_{q} / G_{q} \subset U_{p} / G_{p}$ can be lifted to a smooth map

$$
\tilde{i}_{p q}: U_{q} \rightarrow U_{p}
$$

and there is an injective homomorphism

$$
i_{\#, p q}: G_{q} \rightarrow G_{p}
$$

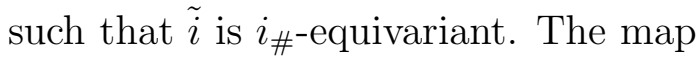

$$
i_{p q}=\left(\tilde{i}, i_{\#}\right):\left(U_{q}, G_{q}\right) \rightarrow\left(U_{p}, G_{p}\right)
$$

is called an injection. A different lifting differs from $\tilde{i}$ by the action of an element of $G_{p}$. Moreover, $i_{\#}$ differs by conjugation by the same element; we say that the corresponding injections are equivalent. Therefore, for any $g \in G_{q}$, the conjugacy class $\left(i_{\#}(g)\right)_{G_{p}}$ is well-defined. Intuitively, we "patch" the conjugacy class $(g)_{G_{q}}$ to the conjugacy class $\left(i_{\#}(g)\right)_{G_{p}}$. Formally, we define an equivalence relation $(g)_{G_{q}} \cong\left(i_{\#}(g)\right)_{G_{p}}$. Let $T_{1}$ be the set of equivalence classes. By abuse of notation, we use $(g)$ to denote the equivalence class that contains $(g)_{G_{q}}$. For each $(g) \in T_{1}$, we define a sector

$$
X_{(g)}=\left\{\left(x,\left(g^{\prime}\right)_{G_{x}}\right) \mid g^{\prime} \in G_{x},\left(g^{\prime}\right)_{G_{x}} \in(g)\right\} .
$$

It was shown in $[\mathbf{C R}]$ that $X_{(g)}$ is an orbifold. In accordance with the common convention we call $X_{(g)}$ for $g \neq 1$ a twisted sector, and $X_{(1)}$ non-twisted sector.

Remark 2.1: In general, a sector is not a subset of the orbifold. Instead, there is a "morphism" $X_{(g)} \rightarrow X$ which is finite-to-one.

In the same fashion, we can also define multi-sectors. Define

$$
\widetilde{\Sigma_{k} X}=\left\{\left(x,\left(g_{1}, \ldots, g_{k}\right)_{G_{x}} ; g_{i} \in G_{x}\right\}\right.
$$

where $\left(g_{1}, \ldots, g_{k}\right)_{G_{x}}=\left\{\left(h g_{1} h^{-1}, \ldots, h g_{k} h^{-1}: h \in G_{x}\right\}\right.$ denotes the joint conjugacy class. Again, $\widetilde{\Sigma_{k} X}$ has a natural orbifold structure. Its connected components can be described in the same way. We define an equivalence relation $\left(g_{1}, \ldots, g_{k}\right)_{G_{q}} \cong\left(i_{\#}\left(g_{1}\right), \ldots, i_{\#}\left(g_{k}\right)\right)_{G_{p}}$. Let $T_{k}$ be the set of equivalence classes. By abusing the notation, we use 
$\left(g_{1}, \ldots, g_{k}\right)$ to denote the equivalence class which contains $\left(g_{1}, \ldots, g_{k}\right)_{G_{q}}$. For each $\left(g_{1}, \ldots, g_{k}\right) \in T_{k}$, we define the multi-sector

$$
X_{\left(g_{1}, \ldots, g_{k}\right)}=\left\{\left(x,\left(g_{1}^{\prime}, \ldots, g_{k}^{\prime}\right)_{G_{x}}\right) \mid g_{i}^{\prime} \in G_{x},\left(g_{1}^{\prime}, \ldots, g_{k}^{\prime}\right)_{G_{x}} \in\left(g_{1}, \ldots, g_{k}\right)\right\} .
$$

2.2. Orbifold vector bundles and orbifold morphisms. We define the notion of orbifold vector bundle $E \rightarrow X$ similarly. An orbifold chart of $E$ over $U_{p} / G_{p}$ is of the form $\left(U_{p} \times \mathbf{R}^{n}, G_{p}\right)$ where $\left(U_{p}, G_{p}\right)$ is an orbifold chart at $p \in X$ and $G_{p}$ acts on $\mathbf{R}^{n}$ linearly. Thus we assume that the total space and base space have the same local groups. The patching condition is as follows. To each injection $i$ on the base, we assign an injection $\lambda(i)$ on the total space such that

$$
\lambda(j \circ i)=\lambda(j) \circ \lambda(i)
$$

for any composition $j \circ i$ of injections on the base orbifold. Without this condition, $\lambda(j \circ i)$ could differ from $\lambda(j) \circ \lambda(i)$ by conjugation with an element of the local group.

Once we understand condition (2.6), it is not hard to see that the the orbifold map (as usually defined) fails to pull back an orbifold vector bundle and a stronger condition has to be imposed. In $[\mathbf{C R}]$, ChenRuan formulated a condition and called it a "good map". Later, it became clear that a good map is equivalent to an earlier but more abstract concept of "orbifold morphism" from the theory of groupoids and we now adopt this name. The definition of $[\mathbf{C R}]$ was formulated with the explicit goal to pull back the vector bundle, and has the advantage that it is concrete and easier to work with in geometry. It follows.

An orbifold morphism is a continuous map $f: X \rightarrow Y$ on the underlying orbifolds with an isomorphism class of extra structure called a compatible system. A compatible system is (i) a one-to-one assignment of orbifold charts on $Y$ to charts on $X$ such that $f$ is locally lifted to an equivariant map with the appropriate diagrams commuting, and (ii) a one-to-one assignment of injections $i \rightarrow \mu(i)$ satisfying the compatibility condition

$$
\mu(j \circ i)=\mu(j) \circ \mu(i) .
$$

It is obvious that an orbifold morphism pulls back an orbifold vector bundle constructed by first pulling back the local orbifold bundles and then gluing them up using condition (2.7).

Recall that the pull-back bundle can be defined by using the fiber product

$$
E \times_{Y} X=\{(v, x) ; \pi(v)=f(x)\} .
$$


In the orbifold category, the ordinary fiber product does not yield an orbifold vector bundle. However, the process of (i) constructing the ordinary fiber product locally over the uniformizing systems, and (ii) then gluing them together using the compatibility condition of the morphism can be viewed as an orbifold version of the fiber product. Without the risk of confusion, we still denote it by $E \times_{Y} X$.

Orbifold morphisms exist abundantly. All the natural maps such as the projections and sections of orbifold vector bundles are orbifold morphisms. A composition of orbifold morphisms is an orbifold morphism, so orbifolds and orbifold morphisms form a category. An important class of orbifold morphisms is defined in the following

Lemma 2.2: The evaluation maps

$$
e_{i_{1}, \ldots, i_{l}}: \widetilde{\Sigma_{k} X} \rightarrow \widetilde{\Sigma_{l} X}
$$

defined as $e_{i_{1}, \ldots, i_{l}}\left(x,\left(g_{1}, \ldots, g_{k}\right)_{G_{x}}\right)=\left(x,\left(g_{i_{1}}, \ldots, g_{i_{l}}\right)_{G_{x}}\right)$, and the involution

$$
I: \widetilde{\Sigma_{k} X} \rightarrow \widetilde{\Sigma_{k} X}
$$

defined by $I\left(x,\left(g_{1}, \ldots, g_{k}\right)_{G_{x}}\right)=\left(x,\left(g_{1}^{-1}, \ldots, g_{k}^{-1}\right)_{G_{x}}\right)$ are orbifold morphisms.

The proof is immediate from the definition since we can construct the orbifold charts and injections of $\widetilde{\Sigma_{k} X}$ simultaneously.

2.3. The orbifold cohomology group and its Poincaré pairing. For a sector $X_{(g)}$ as in (2.3) we diagonalize the action of $g$ in $T_{e(x)} X$ for each $x \in X_{(g)}$, where $e: X_{(g)} \rightarrow X$ is the evaluation map. Suppose that $g=\operatorname{diag}\left(e^{\frac{2 \pi i m_{1}}{m}}, \ldots, e^{\frac{2 \pi i m_{n}}{m}}\right)$, where $m$ is the order of $g$ and $0 \leq \frac{m_{i}}{m}<1$. Then we define the degree shifting number

$$
\iota_{(g)}=\sum_{i} \frac{m_{i}}{m}
$$

It can be shown that $\iota_{(g)}$ is independent of $x \in X_{(g)}$. The ordinary orbifold cohomology is defined as

$$
H_{o r b}^{*}(X, \mathbf{C})=\bigoplus_{(g) \in T_{1}} H^{*-2 \iota(g)}\left(X_{(g)}, \mathbf{C}\right) .
$$

Recall the involution $I: X_{(g)} \rightarrow X_{\left(g^{-1)}\right.}$ defined by $(x,(g)) \rightarrow\left(x,\left(g^{-1}\right)\right)$. The Poincaré pairing \langle\rangle$_{\text {orb }}$ of orbifold cohomology is the direct sum over $(g) \in T_{1}$ of the bilinear maps

$$
\langle\rangle_{\text {orb }}^{(g)}: H^{d-2 \iota(g)}\left(X_{(g)}, \mathbf{C}\right) \otimes H^{2 n-d-2 \iota_{(g-1)}}\left(X_{\left(g^{-1}\right)}, \mathbf{C}\right) \rightarrow \mathbf{C}
$$


defined by

$$
\langle\alpha, \beta\rangle_{\text {orb }}^{(g)}=\int_{X_{(g)}} \alpha \wedge I^{*} \beta
$$

Remark 2.3: There is a compactly supported version of the orbifold cohomology defined in the similar fashion. When $X$ is open, Poincaré duality should be understood as a pairing between the ordinary orbifold cohomology and the compactly supported orbifold cohomology.

2.4. The orbifold cup product. Recall the homological algebra notion $H^{p}().[i]=H^{p+i}($.$) . For \quad \alpha \in H^{p}\left(X_{\left(g_{1}\right)} \mathbf{R}\right)\left[-2 \iota_{\left(g_{1}\right)}\right]$ and $\beta \in H^{q}(X, \mathbf{R})\left[-2 \iota_{\left(g_{2}\right)}\right]$ define $\alpha \cup_{\text {orb }} \beta \in H_{\text {orb }}^{p+q}(X, \mathbf{R})$ by

$$
\alpha \cup_{\text {orb }} \beta=\sum_{\left(h_{1}, h_{2}\right) \in T_{2}, h_{i} \in\left(g_{i}\right)}\left(\alpha \cup_{\text {orb }} \beta\right)_{\left(h_{1}, h_{2}\right)},
$$

where $\left(\alpha \cup_{\text {orb }} \beta\right)_{\left(h_{1}, h_{2}\right)} \in H^{*}\left(X_{\left(h_{1} h_{2}\right)}, \mathbf{C}\right)$ is defined by the relation

$$
\left\langle\left(\alpha \cup_{\text {orb }} \beta\right)_{\left(h_{1}, h_{2}\right)}, \gamma\right\rangle_{\text {orb }}=\int_{X_{\left(h_{1}, h_{2}\right)}} e_{1}^{*} \alpha \wedge e_{2}^{*} \beta \wedge e_{3}^{*} \gamma \wedge e_{A}\left(E_{\left(h_{1}, h_{2}\right)}\right) \text {. }
$$

Each term of formula (2.12) is defined as follows. $\gamma \in H_{c}^{*}\left(X_{\left(\left(h_{1} h_{2}\right)^{-1}\right)}, \mathbf{C}\right)$. $e_{1}, e_{2}, e_{3}$ are the evaluation maps

$$
\begin{gathered}
e_{1}\left(x,\left(h_{1}^{\prime}, h_{2}^{\prime}\right)\right)=\left(x,\left(h_{1}^{\prime}\right)\right), \quad e_{2}\left(x,\left(h_{1}^{\prime}, h_{2}^{\prime}\right)\right)=\left(x,\left(h_{2}^{\prime}\right)\right), \\
e_{3}\left(x,\left(h_{1}^{\prime}, h_{2}^{\prime}\right)\right)=\left(x,\left(h_{1}^{\prime} h_{2}^{\prime}\right)^{-1}\right),
\end{gathered}
$$

and $e_{A}\left(E_{\left(h_{1}, h_{2}\right)}\right)$ is the Euler class of the obstruction bundle $E_{\left(h_{1}, h_{2}\right)}$ defined below.

Remark 2.4: In the case of a global quotient $X=Y / G, X_{\left(h_{1}, h_{2}\right)}=$ $Y_{h_{1}} \cap Y_{h_{2}} / C\left(h_{1}\right) \cap C\left(h_{2}\right)$.

The construction of the obstruction bundle $E_{\left(h_{1}, h_{2}\right)}$ is motivated by the genus zero degree zero orbifold Gromov-Witten invariants (see $[\mathbf{C R}])$. The construction is very concrete and can be written down explicitly without referring to GW-theory.

Let $G_{0}=\left\langle h_{1}, h_{2}\right\rangle$ be the subgroup of the local group of $X_{\left(h_{1}, h_{2}\right)}$ generated by $h_{1}, h_{2}$. Suppose that $k_{1}=\operatorname{ord}\left(h_{1}\right), k_{2}=\operatorname{ord}\left(h_{2}\right), k_{3}=$ $\operatorname{ord}\left(\left(h_{1} h_{2}\right)^{-1}\right)$. There is an orbifold Riemann sphere $S$ with three orbifold points with local groups $\mathbf{Z} / k_{1}, \mathbf{Z} / k_{2}, \mathbf{Z} / k_{3}$. Its orbifold fundamental group is

$$
\pi_{1}^{\text {orb }}(S)=\left\{\lambda_{1}, \lambda_{2}, \lambda_{3} \mid \lambda_{i}^{k_{i}}=1(i \leq 3), \quad \lambda_{1} \lambda_{2} \lambda_{3}=1\right\} .
$$


There is an obvious surjective homomorphism $\pi_{1}^{\text {orb }}(S) \rightarrow G_{0}$ by mapping $\lambda_{1} \rightarrow h_{1}, \lambda_{2} \rightarrow h_{2}, \lambda_{3} \rightarrow\left(h_{1} h_{2}\right)^{-1}$. Using the theory of orbifold covers, we obtain a smooth compact Riemann surface $\Sigma$ such that $G_{0}$ acts on $\Sigma$ and $\Sigma / G_{0}=S$ as orbifolds.

Let $e_{\left(h_{1}, h_{2}\right)} \rightarrow X$ be the evaluation map. It is easy to check that $G_{0}$ acts on the total space of $e_{\left(h_{1}, h_{2}\right)}^{*} T X$ linearly. The obstruction bundle $E_{\left(h_{1}, h_{2}\right)}$ is defined as

$$
E_{\left(h_{1}, h_{2}\right)}=\left(H^{0,1}(\Sigma) \otimes e_{\left(h_{1}, h_{2}\right)}^{*} T X\right)^{G_{0}} .
$$

Remark 2.5: There is a Dolbeault version of the orbifold cohomology ring (Dolbeault orbifold cohomology ring) with the identical construction. We refer reader to $[\mathbf{C R}]$ for details.

\section{Inner local systems and the twisted orbifold cohomology ring}

Recall that for $\left(h_{1}, \cdots, h_{k}\right) \in T_{k}$, there are $k+1$ evaluation maps

$$
e_{i}: X_{\left(h_{1}, \cdots, h_{k}\right)} \rightarrow X_{\left(h_{i}\right)}, i \leq k,
$$

and

$$
e_{k+1}: X_{\left(h_{1}, \cdots, h_{k}\right)} \rightarrow X_{\left(h_{1} \cdots h_{k}\right)}
$$

Now, we introduce the notion of inner local system for an orbifold.

Definition 3.1: Suppose that $X$ is an orbifold (almost complex or not). An inner local system $\mathbf{L}=\left\{L_{(g)}\right\}_{g \in T_{1}}$ is an assignment of a flat complex orbifold line bundle

$$
L_{(g)} \rightarrow X_{(g)}
$$

to each sector $X_{(g)}$ satisfying the following compatibility conditions (1-4).

(1): $L_{(1)}$ is a trivial orbifold line bundle with a fixed trivialization.

(2): There is a nondegenerate pairing $L_{(g)} \otimes I^{*} L_{\left(g^{-1}\right)} \rightarrow \mathbf{C}=L_{(1)}$.

(3): There is a multiplication

$$
e_{1}^{*} L_{\left(h_{1}\right)} \otimes e_{2}^{*} L_{\left(h_{2}\right)} \stackrel{\theta}{\rightarrow} e_{3}^{*} L_{\left(h_{1} h_{2}\right)}
$$

over $X_{\left(h_{1}, h_{2}\right)}$ for $\left(h_{1}, h_{2}\right) \in T_{2}$.

(4): $\theta$ is associative in the following sense. For $\left(h_{1}, h_{2}, h_{3}\right) \in T_{3}$, the evaluation maps $e_{i}: X_{\left(h_{1}, h_{2}, h_{3}\right)} \rightarrow X_{\left(h_{i}\right)}$ factor through

$$
P=\left(P_{1}, P_{2}\right): X_{\left(h_{1}, h_{2}, h_{3}\right)} \rightarrow X_{\left(h_{1}, h_{2}\right)} \times X_{\left(h_{1} h_{2}, h_{3}\right)} .
$$


Let $e_{12}: X_{\left(h_{1}, h_{2}, h_{3}\right)} \rightarrow X_{\left(h_{1} h_{2}\right)}$. We first use $P_{1}$ to define

$$
\theta: e_{1}^{*} L_{\left(h_{1}\right)} \otimes e_{2}^{*} L_{\left(h_{2}\right)} \rightarrow e_{12}^{*} L_{\left(h_{1} h_{2}\right)} .
$$

Then, we can use $P_{2}$ to define a product

$$
\theta: e_{12}^{*} L_{\left(h_{1} h_{2}\right)}^{*} \otimes L_{\left(h_{3}\right)}^{*} \rightarrow e_{4}^{*} L_{\left(h_{1} h_{2} h_{3}\right)}^{*} .
$$

Taking the composition, we define

$$
\theta\left(\theta\left(e_{1}^{*} L_{\left(h_{1}\right)}, e_{2}^{*} L_{\left(h_{2}\right)}\right), e_{3}^{*} L_{\left(h_{3}\right)}\right): e_{1}^{*} L_{\left(h_{1}\right)} \otimes e_{2}^{*} L_{\left(h_{2}\right)} \otimes e_{3}^{*} L_{\left(h_{3}\right)} \rightarrow e_{4}^{*} L_{\left(h_{4}\right)}^{*} .
$$

On the other hand, the evaluation maps $e_{i}$ also factor through

$$
P^{\prime}: X_{\left(h_{1}, h_{2}, h_{3}\right)} \rightarrow X_{\left(h_{1}, h_{2} h_{3}\right)} \times X_{\left(h_{2}, h_{3}\right)} .
$$

In the same way, we can define another triple product

$\operatorname{theta}\left(e_{1}^{*} L_{\left(h_{1}\right)}, \theta\left(e_{2}^{*} L_{\left(h_{2}\right)}, e_{3}^{*} L_{\left(h_{3}\right)}\right)\right): e_{1}^{*} L_{\left(h_{1}\right)} \otimes e_{2}^{*} L_{\left(h_{2}\right)} \otimes e_{3}^{*} L_{\left(h_{3}\right)} \rightarrow e_{4}^{*} L_{\left(h_{4}\right)}^{*}$.

Then, we require the associativity

$$
\theta\left(\theta\left(e_{1}^{*} L_{\left(h_{1}\right)}, e_{2}^{*} L_{\left(h_{2}\right)}\right), e_{3}^{*} L_{\left(h_{3}\right)}\right)=\theta\left(e_{1}^{*} L_{\left(h_{1}\right)}, \theta\left(e_{2}^{*} L_{\left(h_{2}\right)}, e_{3}^{*} L_{\left(h_{3}\right)}\right)\right) .
$$

If $X$ is a complex orbifold, we assume that $L_{(g)}$ is holomorphic.

Definition 3.2: Given an inner local system $\mathbf{L}$, we define the twisted orbifold cohomology

$$
H_{\text {orb }}^{*}(X, \mathbf{L})=\bigoplus_{(g)} H^{*-2 \iota_{(g)}}\left(X_{(g)}, L_{(g)}\right) .
$$

Definition 3.3: Suppose that $X$ is a closed complex orbifold and $\mathbf{L}$ is an inner local system. We define Dolbeault cohomology groups

$$
H_{o r b}^{p, q}(X, \mathbf{L})=\bigoplus_{(g)} H^{p-\iota(g), q-\iota(g)}\left(X_{(g)} ; L_{(g)}\right) .
$$

Proposition 3.4: If $X$ is a Kähler orbifold, we have the Hodge decomposition

$$
H_{o r b}^{k}(X, \mathbf{L})=\bigoplus_{k=p+q} H_{o r b}^{p, q}(X, \mathbf{L})
$$

Proof: Note that each sector $X_{(g)}$ is a Kähler orbifold. The proposition follows by applying the ordinary Hodge theorem with twisted coefficients to each sector $X_{(g)}$.

The property (2) of Definition 3.1 can be used to show that the Poincaré pairing defined in section 2.3 can be adapted to twisted orbifold cohomology. 
Definition (Poincaré duality) 3.5: Suppose that $X$ is a $2 n$ dimensional closed almost complex orbifold. We define a pairing

$$
\langle\rangle_{o r b, \mathbf{L}}: H_{o r b}^{d}(X, \mathbf{L}) \otimes H_{o r b}^{2 n-d}(X, \mathbf{L}) \rightarrow \mathbf{C} .
$$

as the direct sum of

$$
\langle\rangle_{o r b, \mathbf{L}}^{(g)}: H^{d-2 \iota_{(g)}}\left(X_{(g)}, L_{(g)}\right) \otimes H^{2 n-d-2 \iota_{\left(g^{-1}\right)}}\left(X_{\left(g^{-1}\right)}, L_{\left(g^{-1}\right)}\right) \rightarrow \mathbf{C}
$$

defined by

$$
\langle\alpha, \beta\rangle_{o r b, \mathbf{L}}^{(g)}=\int_{X_{(g)}} \alpha \wedge I^{*} \beta .
$$

Note that $L_{(g)} I^{*} L_{\left(g^{-1}\right)}=1$. Hence the integral (3.5) makes sense.

Theorem 3.6: The pairing \langle\rangle$_{o r b, \mathbf{L}}$ is nondegenerate.

Proof: This follows from ordinary Poincaré duality on $X_{(g)}$ with twisted coefficients.

There is also a version of Poincaré duality for twisted Dolbeault cohomology. Suppose that $X$ is a closed complex orbifold of complex dimension $n$. Then $X_{(g)}$ is a closed complex orbifold.

Definition 3.7: We define a pairing

$$
\langle\rangle_{o r b, \mathbf{L}}: H_{o r b}^{p, q}(X, \mathbf{L}) \otimes H_{o r b}^{n-p, n-q}(X, \mathbf{L}) \rightarrow \mathbf{C} .
$$

as the direct sum of

\langle\rangle$_{\text {orb }, \mathbf{L}}^{(g)}: H^{p-\iota_{(g)}, q-\iota(g)}\left(X_{(g)}, L_{(g)}\right) \otimes H^{n-p-\iota\left(g^{-1}\right), n-q-\iota}\left(g^{-1}\right)\left(X_{\left(g^{-1}\right)}, L_{\left(g^{-1}\right)}\right) \rightarrow \mathbf{C}$ defined by

$$
\langle\alpha, \beta\rangle_{\text {orb }, \mathbf{L}}^{(g)}=\int_{X_{(g)}} \alpha \wedge I^{*} \beta .
$$

Theorem 3.8: The pairing (3.6) is nondegenerate.

Using property (2), we can identify $I^{*} L_{\left(g^{-1}\right)} \cong L_{(g)}^{*}$. There is a canonical isomorphism

$$
P: X_{\left(h_{1}, h_{2}\right)} \rightarrow X_{\left(h_{1}, h_{2},\left(h_{1} h_{2}\right)^{-1}\right)} .
$$

Note that $e_{3} \circ P=I \circ e_{3}$. Applying the above identifications to $\theta$, we obtain a triple pairing

$$
\bar{\theta}: e_{1}^{*} L_{\left(h_{1}\right)} \otimes e_{2}^{*} L_{\left(h_{2}\right)} \otimes e_{2}^{*} L_{\left(h_{1} h_{2}\right)^{-1}} \rightarrow \mathbf{C} .
$$


$\bar{\theta}$ is the property needed to show that the integral (2.12) makes sense for twisted orbifold cohomology classes. Hence, we can define a twisted orbifold product $\cup_{\text {orb }, \mathbf{L}}$. By checking the proof of associativity in $[\mathbf{C R}]$, one can easily show that the associativity of $\theta$ is precisely the property need to prove associativity of the twisted orbifold product $\cup_{\text {orb } \mathbf{L}}$.

Theorem 3.9: $\quad$ Let $X$ be a closed almost complex orbifold with almost complex structure $J$ and $\operatorname{dim}_{\mathbf{C}} X=n$. There is a cup product $\cup_{o r b, \mathbf{L}}: H_{\text {orb }}^{p}(X ; \mathbf{L}) \times H_{o r b}^{q}(X ; \mathbf{L}) \rightarrow H_{o r b}^{p+q}(X ; \mathbf{L})$ for any $0 \leq p, q \leq 2 n$ such that $p+q \leq 2 n$, which has the following properties:

1) The total twisted orbifold cohomology group

$$
H_{o r b}^{*}(X ; \mathbf{L})=\bigoplus_{0 \leq d \leq 2 n} H_{o r b}^{d}(X ; \mathbf{L})
$$

is a ring with unit $e_{X}^{0} \in H_{\text {orb }}^{0}(X ; \mathbf{L})$ under $\cup_{\text {orb }, \mathbf{L}}$, where $e_{X}^{0}$ is the Poincaré dual to the fundamental class of the nontwisted sector.

2) Restricted to each $H_{\text {orb }}^{d}(X ; \mathbf{L}) \times H_{\text {orb }}^{2 n-d}(X ; \mathbf{L}) \rightarrow H_{\text {orb }}^{2 n}(X ; \mathbf{L})=$ $H^{2 n}(X, \mathbf{C})$,

$$
\int_{X} \alpha \cup_{o r b, \mathbf{L}} \beta=\langle\alpha, \beta\rangle_{o r b, \mathbf{L}} .
$$

3) The cup product $\cup_{\text {orb }, \mathbf{L}}$ is invariant under deformations of $J$.

4) When $X$ is of integral degree shifting numbers, the total twisted orbifold cohomology group $H_{\text {orb }}^{*}(X ; \mathbf{L})$ is integrally graded, and we have supercommutativity

$$
\alpha_{1} \cup_{o r b, \mathbf{L}} \alpha_{2}=(-1)^{\operatorname{deg} \alpha_{1} \cdot \operatorname{deg} \alpha_{2}} \alpha_{2} \cup_{o r b, \mathbf{L}} \alpha_{1} .
$$

5) Restricted to the nontwisted sectors, i.e., the ordinary cohomology $H^{*}(X ; \mathbf{C})$, the cup product $\cup_{o r b, \mathbf{L}}$ equals the ordinary cup product on $X$.

6) $\cup_{o r b, \mathbf{L}}$ is associative.

Similarly, we also have a holomorphic version.

Theorem 3.10: Let $X$ be an n-dimensional closed complex orbifold with complex structure $J$. The orbifold cup product

$$
\cup_{o r b, \mathbf{L}}: H_{o r b}^{p, q}(X ; \mathbf{L}) \times H_{o r b}^{p^{\prime}, q^{\prime}}(X ; \mathbf{L}) \rightarrow H_{o r b}^{p+p^{\prime}, q+q^{\prime}}(X ; \mathbf{L})
$$

has the following properties:

1) The total orbifold Dolbeault cohomology group is a ring with unit $e_{X}^{0} \in H_{o r b}^{0,0}(X ; \mathbf{L})$ under $\cup_{o r b, \mathbf{L}}$, where $e_{X}^{0}$ is the class represented by the constant 1 function on $X$. 
2) Restricted to each $H_{o r b}^{p, q}(X ; \mathbf{L}) \times H_{o r b}^{n-p, n-q}(X ; \mathbf{L}) \rightarrow H_{o r b}^{n, n}(X ; \mathbf{L})$, the integral $\int_{X} \alpha \cup_{o r b, \mathbf{L}} \beta$ equals the Poincaré pairing $\langle\alpha, \beta\rangle_{\text {orb }, \mathbf{L}}$.

3) The cup product $\cup_{o r b, \mathbf{L}}$ is invariant under deformation of $J$.

4) When $X$ is of integral degree shifting numbers, the total twisted orbifold Dolbeault cohomology group of $X$ is integrally graded, and we have supercommutativity

$$
\alpha_{1} \cup_{o r b, \mathbf{L}} \alpha_{2}=(-1)^{\operatorname{deg} \alpha_{1} \cdot \operatorname{deg} \alpha_{2}} \alpha_{2} \cup_{o r b, \mathbf{L}} \alpha_{1} .
$$

5) Restricted to the nontwisted sectors, i.e., the ordinary Dolbeault cohomologies $H^{*, *}(X ; \mathbf{C})$, the cup product $\cup_{o r b, \mathbf{L}}$ equals the ordinary wedge product on $X$.

6) The cup product is associative.

7) When $X$ is Kähler, the cup product $\cup_{o r b, \mathbf{L}}$ coincides with the twisted orbifold cup product over the twisted orbifold cohomology groups $H_{\text {orb }}^{*}(X ; \mathbf{L})$ under the relation

$$
H_{o r b}^{r}(X ; \mathbf{L}) \otimes \mathbf{C}=\bigoplus_{p+q=r} H_{o r b}^{p, q}(X ; \mathbf{L})
$$

Remark 3.11: If $X$ is open, we can define the usual twisted orbifold cohomology $H_{\text {orb }}^{*}(X, \mathbf{L})$ and twisted orbifold cohomology with compact support $H_{o r b, c}^{*}(X, \mathbf{L})$ in the same fashion. The Poincaré pairing should be understood as the pairing between $H_{o r b}^{d}(X, \mathbf{L})$ and $H_{o r b, c}^{2 n-d}(X, \mathbf{L})$.

\section{Orbifold fundamental group and discrete torsion}

First, we recall the definition of orbifold fundamental group.

Definition 4.1: A smooth map $f: Y \rightarrow X$ is an orbifold cover iff (1) each $p \in Y$ has a neighborhood $U_{p} / G_{p}$ such that the restriction of $f$ to $U_{p} / G_{p}$ is isomorphic to a map $U_{p} / G_{p} \rightarrow U_{p} / \Gamma$ where $G_{p} \subset \Gamma$ is a subgroup. (2) Each $q \in X$ has a neighborhood $U_{q} / G_{q}$ for which each component of $f^{-1}\left(U_{q} / G_{q}\right)$ is isomorphic to $U_{q} / \Gamma^{\prime}$ such that $\Gamma^{\prime} \subset G_{q}$ is a subgroup. An orbifold universal cover is an orbifold cover $f: Y \rightarrow X$ of $X$ satisfying the properties (i) $Y$ is connected; (ii) if $f^{\prime}: Y^{\prime} \rightarrow X$ is an orbifold cover, then there exists an orbifold cover $h: Y \rightarrow Y^{\prime}$ such that $f=f^{\prime} \circ h$. If $Y$ exists, we call $Y$ the orbifold universal cover of $X$ and the group of deck translations the orbifold fundamental group $\pi_{1}^{\text {orb }}(X)$ of $X$.

By Thurston $[\mathbf{T}]$, an orbifold universal cover exists. It is clear from the definition that the orbifold universal cover is unique. Suppose that 
$f: Y \rightarrow X$ is an orbifold universal cover. Then

$$
f: Y-f^{-1}(\Sigma X) \rightarrow X-\Sigma X
$$

is a cover in the usual sense with $G=\pi_{1}^{\text {orb }}(X)$ as covering group, where $\Sigma X$ is the singular locus of $X$. Therefore, $X=Y / G$ and there is a surjective homomorphism

$$
p_{f}: \pi_{1}(X-\Sigma X) \rightarrow G .
$$

(It does not follow that (4.1) is a universal cover, and so $p_{f}$ need not be an isomorphism.)

Remark 4.2: $\quad$ Suppose that $X=Z / G$ for an orbifold $Z$ and $Y$ is the orbifold universal cover of $Z$. By the definition, $Y$ is an orbifold universal cover of $X$. It is clear that there is a short exact sequence

$$
1 \rightarrow \pi_{1}(Z) \rightarrow \pi^{\text {orb }}(X) \rightarrow G \rightarrow 1 \text {. }
$$

Example 4.3: Consider the Kummer surface $T^{4} / \tau$ where $\tau$ is the involution

$$
\tau\left(e^{i t_{1}}, e^{i t_{2}}, e^{i t_{3}}, e^{i t_{4}}\right)=\left(e^{-i t_{1}}, e^{-i t_{2}}, e^{-i t_{3}}, e^{-i t_{4}}\right) .
$$

The universal cover is $\mathbf{R}^{4}$. The group $G$ of deck translations is generated by translations $\lambda_{i}$ by an integral point and the involution

$$
\tau:\left(t_{1}, t_{2}, t_{3}, t_{4}\right) \rightarrow\left(-t_{1},-t_{2},-t_{3},-t_{4}\right) .
$$

It is easy to check that

$$
G=\left\{\lambda_{i}(i=1,2,3,4), \tau \mid \tau^{2}=1, \tau \lambda_{i}=\lambda_{i}^{-1} \tau,\right\}
$$

where $\lambda_{i}$ represents translation and $\tau$ represents involution.

Example 4.4: Let $T^{6}=\mathbf{R}^{6} / \Gamma$ where $\Gamma$ is the lattice of integral points. Consider $\mathbf{Z}_{2}^{2}$ acting on $T^{6}$ lifted to an action on $\mathbf{R}^{6}$ as

$$
\begin{gathered}
\sigma_{1}\left(t_{1}, t_{2}, t_{3}, t_{4}, t_{5}, t_{6}\right)=\left(-t_{1},-t_{2},-t_{3},-t_{4}, t_{5}, t_{6}\right) \\
\sigma_{2}\left(t_{1}, t_{2}, t_{3}, t_{4}, t_{5}, t_{6}\right)=\left(-t_{1},-t_{2}, t_{3}, t_{4},-t_{5},-t_{6}\right) \\
\sigma_{3}\left(t_{1}, t_{2}, t_{3}, t_{4}, t_{5}, t_{6}\right)=\left(t_{1}, t_{2},-t_{3},-t_{4},-t_{5}, t_{6}\right) .
\end{gathered}
$$

This example was considered by Vafa-Witten $[\mathbf{V W}]$. The orbifold fundamental group

$$
\pi_{1}^{\text {orb }}\left(T^{6} / \mathbf{Z}_{2}^{2}\right)=
$$

(4.6) $\left\{\tau_{i}(1 \leq i \leq 6), \sigma_{j}(1 \leq j \leq 3) \mid \sigma_{i}^{2}=1, \sigma_{1} \tau_{i}=\tau_{i}^{-1} \sigma_{1}(i \neq 5,6)\right.$,

$$
\left.\sigma_{2} \tau_{i}=\tau_{i}^{-1} \sigma_{2}(i \neq 3,4), \sigma_{3} \tau_{i}=\tau_{i}^{-1} \sigma_{3}(i \neq 1,2)\right\} \text {. }
$$


Example 4.5: Consider the orbifold Riemann surface $\Sigma_{g}$ of genus $g$ and $n$ orbifold points $\mathbf{z}=\left(x_{1}, \ldots, x_{n}\right)$ with orders $k_{1}, \ldots, k_{n}$. Then,

$$
\pi_{1}^{o r b}\left(\Sigma_{g}\right)=\left\{\lambda_{i}(i \leq 2 g), \sigma_{i}(i \leq n) \mid \sigma_{1} \ldots \sigma_{n} \prod_{i}\left[\lambda_{2 i-1}, \lambda_{2 i}\right]=1, \sigma_{i}^{k_{i}}=1\right\}
$$

where $\lambda_{i}$ are the generators of $\pi_{1}\left(\Sigma_{g}\right)$ and $\sigma_{i}$ are the generators of $\Sigma_{g}-\mathbf{z}$ represented by a loop around each orbifold point. Note that $\pi_{1}^{\text {orb }}\left(\Sigma_{g}\right)$ is just $\pi_{1}\left(\Sigma_{g}-\mathbf{z}\right)$ modulo the relation $\sigma_{i}^{k_{i}}=1$. This suggests that one can first take the cover of $\Sigma_{g}-\mathbf{z}$ induced by $\pi_{1}^{\text {orb }}(\Sigma)$. The relation $\sigma_{i}^{k_{i}}=1$ implies that the preimage of the punctured disc around $x_{i}$ is a punctured disc. Then we can fill in the center point to obtain the orbifold universal cover. (This example was taken from $[\mathbf{S C}]$.)

Definition 4.6: A discrete torsion $\alpha$ is defined as a cohomology class $\alpha \in H^{2}\left(\pi_{1}^{\text {orb }}(X), U(1)\right)$.

If $X=Z / G$ for a finite group $G$, by Remark 3.2, there is a surjective homomorphism

$$
\pi: \pi_{1}^{\text {orb }}(X) \rightarrow G
$$

$\pi$ induces a homomorphism

$$
\pi^{*}: H^{2}(G, U(1)) \rightarrow H^{2}\left(\pi_{1}^{\text {orb }}(X), U(1)\right) .
$$

Hence, an element of $H^{2}(G, U(1))$ induces a discrete torsion of $X$.

They are many ways to define $H^{2}(G, U(1))$. The definition $H^{2}(G, U(1))=$ $H^{2}(B G, U(1))$ is a very useful definition for computation since we can use algebro-topological machinery. However, we can also use the original definition in terms of cocycles. A 2-cocycle is a map $\alpha: G \times G \rightarrow$ $U(1)$ satisfying

$$
\alpha_{g, 1}=\alpha_{1, g}=1, \alpha_{g, h k} \alpha_{h, k}=\alpha_{g, h} \alpha_{g h, k},
$$

for any $g, h, k \in G$. We denote the set of 2-cocycles by $Z^{2}(G, U(1))$. For any map $\rho: G \rightarrow U(1)$ with $\rho_{1}=1$, its coboundary is defined by the formula

$$
(\delta \rho)_{g, h}=\rho_{g} \rho_{h} \rho_{g h}^{-1} .
$$

Let $B^{2}(G, U(1))$ be the set of coboundaries. Then $H^{2}(G, U(1))=$ $Z^{2}(G, U(1)) / B^{2}(G, U(1))$. $H^{2}(G, U(1))$ naturally appears in many important places of mathematics. For example, it classifies the group extensions of $G$ by $U(1)$. If we have a unitary projective representation of $G$, it naturally induces a class of $H^{2}(G, U(1))$. In many instances, this class completely classifies the projective unitary representation. In 
fact, it is in this context that discrete torsion arises in orbifold string theory.

Definition 4.7: For each 2-cocycle $\alpha$, we define its phase

$$
\gamma(\alpha)_{g, h}=\alpha_{g, h} \alpha_{g h g^{-1}, g}^{-1}
$$

It is clear that $\gamma(\alpha)_{g, g}=1$.

\section{Lemma 4.8:}

(1): $\gamma(\alpha)_{k h, g}=\gamma(\alpha)_{k, h g h^{-1}} \gamma(\alpha)_{h, g}$

(2): Suppose that $g h=h g, g k=k g$. Then $\gamma(\delta \rho)_{g, h}=1, \gamma(\alpha)_{g, h}=$ $\gamma(\alpha)_{h, g}^{-1}$ and $\gamma(\alpha)_{k h, g}=\gamma(\alpha)_{k, g} \gamma(\alpha)_{h, g}$.

Item (2) implies that the map $L_{g}^{\alpha}=\gamma_{\cdot, g}: C(g) \rightarrow U(1)$ is a group homomorphism. We call $L_{g}^{\alpha}$ an $\alpha$-twisted character.

Proof: (2) is an obvious consequence of (1).

$$
\begin{gathered}
\gamma(\alpha)_{k h, g}=\alpha_{k h, g} \alpha_{k h g h^{-1} k^{-1}, k h^{\circ}}^{-1} \\
\alpha_{k h g h^{-1} k^{-1, k h}}^{-1}=\alpha_{k h g h^{-1} k^{-1}, g_{k h g h^{-1}, h}^{-1}} \alpha_{k, h}^{-1} . \\
\alpha_{k h g h^{-1}, h}^{-1}=\alpha_{k, h g}^{-1} \alpha_{k, h g h^{-1}} \alpha_{h g h^{-1}, h}^{-1} .
\end{gathered}
$$

Then, we have

$\alpha_{k h, g} \alpha_{k h g h^{-1} k^{-1}, g}^{-1} \alpha_{k, h g}^{-1} \alpha_{k, h g h^{-1}} \alpha_{h g h^{-1}, h}^{-1} \alpha_{k, h}=\alpha_{k, h g h^{-1}} \alpha_{k h g h^{-1} k^{-1}, g}^{-1} \alpha_{h, g} \alpha_{h g h^{-1}, h}^{-1}$.

Hence,

$$
\gamma(\alpha)_{k h, g}=\gamma(\alpha)_{k, h g h^{-1}} \gamma(\alpha)_{h, g}
$$

Next, we calculate discrete torsions for some groups. We first consider the case of a finite abelian group $G$. In this case $H^{i}(G, \mathbf{Q})=0$ for $i \neq 0$. The exact sequence

$$
0 \rightarrow \mathbf{Z} \rightarrow \mathbf{C} \rightarrow \mathbf{C}^{*} \rightarrow 1
$$

implies that $H^{2}(G, U(1))=H^{2}\left(G, \mathbf{C}^{*}\right)=H^{3}(G, \mathbf{Z})$. By the universal coefficient theorem, $H^{3}(G, \mathbf{Z})=H_{2}(G, \mathbf{Z})$.

Example $4.9 G=\mathbf{Z} / n \times \mathbf{Z} / m$ : Note that $H^{2}(G, U(1))=H_{2}(G, \mathbf{Z})=$ $\mathbf{Z} / n \otimes \mathbf{Z} / m=\mathbf{Z} / \operatorname{gcd}(n, m)$. In this case, one can write down the phase of the discrete torsion explicitly as in $[\mathbf{V W}]$. Let $\xi$ (resp. $\zeta$ ) be an $n$th (resp. $m$ th) root of unity. Any element of $\mathbf{Z} / n \times \mathbf{Z} / m$ can be written as $\left(\xi^{a}, \zeta^{b}\right)$. Let $p=\operatorname{gcd}(n, m)$ and $\omega_{p}=e^{2 \pi i / p}$. Then

$$
\gamma_{\left(\xi^{a}, \zeta^{b}\right),\left(\xi^{a^{\prime}}, \zeta^{b^{\prime}}\right)}=\omega_{p}^{m\left(a b^{\prime}-b a^{\prime}\right)}
$$


is the phase of a discrete torsion for $m=1, \ldots, p$. (There are $p$ phases, each corresponding to a different discrete torsion.) It is trivial to generalize this construction to an arbitrary finite abelian group.

\section{Discrete torsions and inner local systems}

Suppose that $f: Y \rightarrow X$ is an orbifold universal cover and $G$ is the orbifold fundamental group which acts on $Y$ such that $X=Y / G$. For any $1 \neq g \in G, g$ acts on $Y$ as a morphism. We define the orbifold version of the fixed point set $Y_{g}$ of $g$ as a fiber product of the morphisms $I d, g: Y \rightarrow Y$. Then $Y_{g}$ is a smooth suborbifold in the sense that there is an orbifold embedding $Y_{g} \rightarrow Y$. However, $Y_{g}$ need not be a subset of $Y$. Then $X_{(g)}=Y_{g} / C(g)$ is obviously a twisted sector of $X$, where $C(g)$ is the centralizer of $g$. It is clear that $Y_{h^{-1} g h}$ is diffeomorphic to $Y_{g}$ by the action of $h$. Some twisted sectors may not arise in such a fashion. We call this kind of sector a dormant sector.

Let $e: X_{(g)} \rightarrow X$ be the evaluation map. We can view $Y \rightarrow X$ as an orbifold principal $\pi_{1}^{\text {orb }}(X)$ bundle over $X$. Hence, we can pull back $Z=e^{*} Y \rightarrow X_{(g)}$ to an orbifold principal $\pi_{1}^{\text {orb }}(X)$-bundle over $X_{(g)}$. $X_{(g)}$ is dormant iff the $\pi_{1}^{\text {orb }}(X)$-action on $Z$ has no kernel. Moreover, $Z$ is a $\pi_{1}^{\text {orb }}(X)$-invariant suborbifold (possibily disconnected). We call $Z a \pi_{1}^{\text {orb }}(X)$ effective suborbifold. We will treat a dormant sector the same as the nontwisted sector.

Let $\alpha$ be a discrete torsion. For a dormant sector, we will always assign a trivial line bundle. We can use $L_{g}^{\alpha}$ to define a flat complex orbifold line bundle

$$
L_{g}=Y_{g} \times_{L_{g}^{\alpha}} \mathbf{C}
$$

over $X_{(g)}$. We can work on the sector $X_{(g)}=Y_{g} / C(g)$ directly as the older version of this article does. We can also use some of ideas from $[\mathbf{K}]$ to lift everything to the fixed point set $Y_{g}$.

As an orbifold, the inertia orbifold $\sqcup_{(g) \in T_{1}} X_{(g)}$ is the quotient of the disjoint union of $\left(\sqcup_{g \in \pi_{1}^{o r b}(X)} Y_{g}\right)$ and $\pi_{1}^{\text {orb }}(X)$-effective suborbifolds by the action of $\pi_{1}^{\text {orb }}(X)$. The inner local system corresponds to the trivial line bundle $Y_{g} \times \mathbf{C}_{g}$. For the $\pi_{1}^{\text {orb }}(X)$-effective suborbifold $Z$, it is also trivial. We denote its fiber by $\mathbf{C}_{1}$ and treat it the same as the nontwisted sector.

Now, we describe the action of $\pi_{1}^{o r b}(X)$, the pairing and the product. Let $1_{h} \in \mathbf{C}_{h}$ be the identity. We define $g: \mathbf{C}_{h} \rightarrow \mathbf{C}_{g h g^{-1}}$ by $g\left(1_{h}\right)=$ $\gamma_{g, h} 1_{g h g^{-1}}$. To show that it defines an action, we need to check that 
$g_{1} g_{2}\left(1_{h}\right)=g_{1}\left(g_{2}\left(1_{h}\right)\right.$. It follows from Lemma 4.8 that

$$
\gamma_{g_{1} g_{2}, h}=\gamma_{g_{1}, g_{2} h g_{2}^{-1}} \gamma_{g_{2}, h}
$$

The product $\mathbf{C}_{g} \otimes \mathbf{C}_{h} \rightarrow \mathbf{C}$ is defined by $1_{g} \cdot 1_{h}=\alpha_{g, h} 1_{g h}$. The associativity of the product follows from the cocycle condition

$$
\alpha_{g, h} \alpha_{g h, k}=\alpha_{g, h k} \alpha_{h, k}
$$

Note that the product gives $1_{g} \cdot 1_{g^{-1}}=\alpha_{g, g^{-1}} 1_{1}$. This is nondegenerate since $\alpha_{g, g^{-1}} \in S^{1}$.

We still have to check that the product is invariant under the $\pi_{1}^{\text {orb }}(X)$ action,i.e.

$$
g\left(1_{h}\right) \cdot g\left(1_{k}\right)=\alpha_{h, k} g\left(1_{h k}\right)
$$

Using the definition of action, it is equivalent to the formula

$$
\gamma_{g, h} \gamma_{g, k} \alpha_{g h g^{-1}, g k g^{-1}}=\alpha_{h, k} \gamma_{g, h k} .
$$

The above formula is equivalent to

\section{Lemma 5.1:}

$$
\alpha_{g, h} \alpha_{g h g^{-1}, g}^{-1} \alpha_{g, k} \alpha_{g k g^{-1, g}} \alpha_{g h g^{-1}, g k g^{-1}}=\alpha_{h, k} \alpha_{g, h k} \alpha_{g h k g^{-1}, g}^{-1} .
$$

Proof: Recall the cocycle condition

$$
\alpha_{g_{1}, g_{2}} \alpha_{g_{1} g_{2}, g_{3}}=\alpha_{g_{1}, g_{2} g_{3}} \alpha_{g_{2}, g_{3}} .
$$

Using $g_{1}=g, g_{2}=h, g_{3}=k$, we have

$$
\alpha_{g, h} \alpha_{g h, k}=\alpha_{g, h k} \alpha_{h, k} .
$$

Using $g_{1}=g h g^{-1}, g_{2}=g, g_{3}=k$, we have

$$
\alpha_{g h g^{-1}, g}^{-1} \alpha_{g, k} \alpha_{g h g^{-1}, g k}=\alpha_{g h, k} .
$$

Using $g_{1}=g h g^{-1}, g_{2}=g k g^{-1}, g_{3}=g$, we have

$$
\alpha_{g h g^{-1}, g k g^{-1}} \alpha_{g h g^{-1}, g k}^{-1} \alpha_{g k g^{-1}, g}^{-1}=\alpha_{g h k g^{-1}, g}^{-1} .
$$

Multiplying all of them together, we obtain Lemma.

By dividing the action of $\pi_{1}^{\text {orb }}(X)$, we obtain

Theorem 5.2: $\quad \mathbf{L}_{\alpha}=\left\{L_{(g)}\right\}_{(g) \in T_{1}}$ with $\theta$ given by the quotient of product is an inner local system of $X$.

Definition 5.3: Suppose that $\alpha$ is a discrete torsion. We define the twisted orbifold cohomology $H_{o r b, \alpha}^{*}(X, \mathbf{C})=H_{o r b}^{*}\left(X, \mathbf{L}_{\alpha}\right)$. 


\section{Examples}

Only a few examples of global quotients have been computed by physicists (see $[\mathbf{V W}],[\mathbf{D}]$ ). It is still a very important problem to develop a general machinery to compute discrete torsion and twisted orbifold cohomology. Here we compute five examples.

Examples 6.1,6.3 have nontrivial discrete torsion. Example 6.1 is a global quotient and the example 6.2 is a non-global quotient. Example 6.2 has the phenomenon that most of the twisted sectors are dormant sectors. The third example is the Joyce example, where there is no nontrivial discrete torsion. However, there are nontrivial inner local systems. We will compute the twisted orbifold cohomology given by nontrivial inner local systems to match Joyce's desingularizations. Orbifold cohomology is strongly intertwined with group theory. We demonstrate this in the last two examples.

Example 6.1 $T^{4} / \mathbf{Z}_{2} \times \mathbf{Z}_{2}$ : Here $T^{4}=\mathbf{C}^{2} / \Lambda$, where $\Lambda$ is the lattice of integral points. Suppose that $g, h$ are generators of the first and the second factor of $\mathbf{Z}_{2} \times \mathbf{Z}_{2}$. The action of $\mathbf{Z}_{2} \times \mathbf{Z}_{2}$ on $T^{4}$ is defined as

$$
g\left(z_{1}, z_{2}\right)=\left(-z_{1}, z_{2}\right), h\left(z_{1}, z_{2}\right)=\left(z_{1},-z_{2}\right) .
$$

The fixed point locus of $g$ consists of four copies of $T^{2}$. When we divide it by the remaining action generated by $h$, we obtain twisted sectors consisting of four copies of $S^{2}$. The degree shifting number for these twisted sectors is $\frac{1}{2}$. For the same reason, the fixed point locus of $h$ gives twisted sectors consisting of four copies of $S^{2}$ with degree shifting number $\frac{1}{2}$. The fixed point locus of $g h$ consists of sixteen points, which are fixed by the whole group. The degree shifting number of the sixteen points is 1 . An easy calculation shows that the nontwisted sector contributes one generator to the degree 0 and degree 4 orbifold cohomology and two generators to degree 2 orbifold cohomology and no other. Using this information, we can compute the ordinary orbifold cohomology group

$$
b_{\text {orb }}^{0}=b_{\text {orb }}^{4}=1, b_{\text {orb }}^{1}=b_{\text {orb }}^{3}=8, b_{\text {orb }}^{2}=18 .
$$

By example 2.10, $H^{2}\left(\mathbf{Z}_{2} \times \mathbf{Z}_{2}, U(1)\right)=\mathbf{Z}_{2}$. By Remark 2.2, the nontrivial generator of $H^{2}\left(\mathbf{Z}_{2} \times \mathbf{Z}_{2}, U(1)\right)$ induces a discrete torsion $\alpha$. Next, we compute the twisted orbifold cohomology $H_{\text {orb }, \alpha}^{*}\left(T^{4} / \mathbf{Z}_{2} \times \mathbf{Z}_{2}, \mathbf{C}\right)$. Note that $\gamma(\alpha)_{g h, g}=\gamma(\alpha)_{g h, h}=-1$. Hence, the flat orbifold bundles over the twisted sectors given by 16 fixed points of $g h$ are nontrivial. Therefore, they contribute nothing to twisted orbifold cohomology. For 
two-dimensional twisted sectors, we consider a component of the fixed point locus of $g$. By the previous description, it is $T^{2}$. The element $h$ acts on $T^{2}$. Then the twisted sector $S^{2}=T^{2} /\{h\}$. We observe that the flat orbifold line bundle over $S^{2}$ is constructed as $L=T^{2} \times_{L_{g}^{\alpha}} \mathbf{C}$. Hence $H^{*}\left(S^{2}, L\right)$ is isomorphic to the space of invariant cohomology of $T^{2}$ under the action of $h$ twisted by $\gamma(\alpha)_{g}$ as $h(\beta)=\gamma(\alpha)_{g, h} h^{*} \beta$. By example 2.10, $\gamma(\alpha)_{g, h}=-1$. The invariant cohomology is $H^{1}\left(T^{2}, \mathbf{C}\right)$. Using the degree shifting number to shift up its degree, we obtain the twisted orbifold cohomology

$$
b_{o r b, \alpha}^{0}=b_{o r b, \alpha}^{4}=1, \quad b_{o r b, \alpha}^{1}=b_{o r b, \alpha}^{3}=0, \quad b_{o r b, \alpha}^{2}=18 .
$$

Example 6.2 $W P\left(2,2 d_{1}\right) \times W P\left(2,2 d_{2}\right)\left(d_{1}, d_{2}>1,\left(d_{1}, d_{2}\right)=1\right)$ : Here $W P(2,2 d)$ is the weighted projective space of weighted $(2,2 d)$. $W P\left(2,2 d_{1}\right) \times W P\left(2,2 d_{2}\right)$ is not a global quotient unless $d_{1}=d_{2}=1$. In fact, its orbifold universal cover is $W P\left(1, d_{1}\right) \times W P\left(1, d_{2}\right)$ and $W P\left(2,2 d_{1}\right) \times W P\left(2,2 d_{2}\right)=W P\left(1, d_{1}\right) \times W P\left(1, d_{2}\right) / \mathbf{Z}_{2} \times \mathbf{Z}_{2}$. Hence, the orbifold fundamental group is $\mathbf{Z}_{2} \times \mathbf{Z}_{2}$. Therefore, there is a nontrivial discrete torsion $\alpha \in H^{2}\left(\mathbf{Z}_{2} \times \mathbf{Z}_{2}, U(1)\right)$.

Next, we describe the twisted sectors. Suppose that $p=[0,1], q=$ $[1,0] \in W P\left(1, d_{1}\right)$. We also use $p, q$ to denote its image in $W P\left(2,2 d_{1}\right)$. We use $p^{\prime}, q^{\prime}$ to denote the corresponding points in $W P\left(1, d_{2}\right), W P\left(2,2 d_{2}\right)$. The spaces $\{p\} \times W P\left(2,2 d_{2}\right),\left\{p^{\prime}\right\} \times W P\left(2,2 d_{1}\right)$ give rise to two twisted sectors with degree shifting number $\frac{1}{2}$. The spaces $\{q\} \times W P\left(2,2 d_{2}\right),\left\{q^{\prime}\right\} \times$ $W P\left(2,2 d_{1}\right)$ give rise to $2 d_{1}-1,2 d_{2}-1$ twisted sectors with degree shifting numbers $\frac{i}{2 d_{1}}, \frac{j}{2 d_{2}}$ for $1 \leq i \leq 2 d_{1}-1,1 \leq j \leq 2 d_{2}-1$. The spaces $\{p\} \times\left\{p^{\prime}\right\}$ give rise to a twisted sector with degree shifting number 1 . The spaces $\{p\} \times\left\{q^{\prime}\right\}$ give rise to $2 d_{2}-1$ twisted sectors with degree shifting numbers $\frac{1}{2}+\frac{i}{2 d_{2}}$ for $1 \leq i \leq 2 d_{2}-1$. The spaces $\{q\} \times\left\{p^{\prime}\right\}$ give rise to $2 d_{1}-1$ twisted sectors with degree shifting numbers $\frac{1}{2}+\frac{i}{2 d_{1}}$ for $1 \leq i \leq 2 d_{1}-1 .\{q\} \times\left\{q^{\prime}\right\}$ give rise to $4 d_{1} d_{2}-1$ twisted sectors with degree shifting numbers $\frac{i}{2 d_{1}}+\frac{j}{2 d_{2}}$ for all $i, j$ except $(i, j)=(0,0)$. Using this information, we can write down ordinary orbifold cohomology 


$$
\begin{gathered}
b_{\text {orb }}^{0}=b_{\text {orb }}^{4}=1, \quad b_{\text {orb }}^{1}=b_{\text {orb }}^{3}=6, \quad b_{\text {orb }}^{2}=6 \\
b_{\text {orb }}^{\frac{i}{d_{1}}}=b_{\text {orb }}^{\frac{i}{d_{2}}}=1, \quad b_{\text {orb }}^{1+\frac{i}{d_{1}}}=b_{\text {orb }}^{1+\frac{i}{d_{2}}}=3, \quad b_{\text {orb }}^{2+\frac{i}{d_{1}}}=b_{\text {orb }}^{2+\frac{i}{d_{2}}}=2, \\
1 \leq i \leq d_{1}-1,1 \leq j \leq d_{2}-1 \\
b_{\text {orb }}^{\frac{i}{d_{1}}+\frac{j}{d_{2}}}=1, \quad 0 \leq i \leq 2 d_{1}-1, \quad 0 \leq j \leq 2 d_{2}, \quad(i, j) \neq(0,0),\left(d_{1}, d_{2}\right) .
\end{gathered}
$$

Next, we compute $H_{o r b, \alpha}^{*}$. In this example, most of twisted sectors are dormant sectors. To find the nondormant sectors, recall that $W P\left(2,2 d_{1}\right) \times W P\left(2,2 d_{2}\right)=W P\left(1, d_{1}\right) \times W P\left(1, d_{2}\right) / \mathbf{Z}_{2} \times \mathbf{Z}_{2}$. Let $g$ be the generator of the first factor and $h$ be the generator of the second factor. The fixed points of $g$ are $\{p, q\} \times W P\left(1, d_{2}\right)$. We have two non-dormant sectors obtained by dividing by the remaining action generated by $h$. However, $\gamma(\alpha)_{g, h}=-1$. There is no invariant cohomology of $W P\left(1, d_{2}\right)$ under the action of $h$ twisted by $L_{g}^{\alpha}$. Hence, these two nondormant twisted sectors give no contribution to twisted orbifold cohomology. Their degree shifting numbers are 1. For the same reason, $W P\left(1, d_{1}\right) \times\left\{p^{\prime}, q^{\prime}\right\} /(g)$ gives no contribution to twisted orbifold cohomology. The fixed point locus of $g h$ consists of four points which give four nondormant sectors. Again, their degree shifting numbers are 1. As we saw in the last example, their flat orbifold bundles are nontrivial. Hence, they give no contribution to twisted orbifold cohomology. Therefore, the twisted orbifold cohomology is

$$
\begin{gathered}
b_{o r b, \alpha}^{0}=b_{o r b, \alpha}^{4}=1, \quad b_{o r b, \alpha}^{1}=b_{o r b, \alpha}^{3}=2, \quad b_{o r b, \alpha}^{2}=2 \\
b_{o r b, \alpha}^{\frac{i}{d_{1}}}=b_{o r b, \alpha}^{\frac{i}{d_{2}}}=1, \quad b_{\text {orb }, \alpha}^{1+\frac{i}{d_{1}}}=b_{\text {orb }, \alpha}^{1+\frac{i}{d_{2}}}=3, \quad b_{\text {orb }}^{2+\frac{i}{d_{1}}}=b_{\text {orb }, \alpha}^{2+\frac{i}{d_{2}}}=2, \\
\quad 1 \leq i \leq d_{1}-1,1 \leq j \leq d_{2}-1 \\
b_{\text {orb }, \alpha}^{\frac{i}{d_{1}}+\frac{j}{d_{2}}}=1, \quad 0 \leq i \leq 2 d_{1}-1, \quad 0 \leq j \leq 2 d_{2}, \quad(i, j) \neq(0,0),\left(d_{1}, d_{2}\right) .
\end{gathered}
$$

Example 6.3 $T^{6} / \mathbf{Z}_{4}$ : Here, $T^{6}=\mathbf{C}^{3} / \Lambda$, where $\Lambda$ is the lattice of integral points. The generator of $\mathbf{Z}_{4}$ acts on $T^{6}$ as

$$
\kappa:\left(z_{1}, z_{2}, z_{3}\right) \rightarrow\left(-z_{1}, i z_{2}, i z_{3}\right)
$$

This example has been studied by D. Joyce [JO], where he constructed five different desingularizations. However, in this case there is no discrete torsion that induces nontrivial orbifold cohomology. 
First of all, the nontwisted sector contributes one generator to $H_{\text {orb }}^{0,0}, H_{o r b}^{3,3}$, five generators to $H_{o r b}^{1,1}, H_{o r b}^{2,2}$ and two generators to $H_{o r b}^{2,1}, H_{o r b}^{1,2}$. The fixed point locus of $\kappa, \kappa^{3}$ consists of the 16 points

$$
\left\{\left(z_{1}, z_{2}, z_{3}\right)+\Lambda: z_{1} \in\left\{0, \frac{1}{2}, \quad \frac{i}{2}, \frac{1}{2}+\frac{i}{2}\right\}, \quad z_{2}, z_{3} \in\left\{0, \frac{1}{2}+\frac{i}{2}\right\} .\right.
$$

These points are fixed by $\mathbf{Z}_{4}$. Therefore, they generate 32 twisted sectors of which 16 correspond to the conjugacy class $(\kappa)$ and 16 correspond to the conjugacy class $\left(\kappa^{3}\right)$. The sector with conjugacy class $(\kappa)$ has degree shifting number 1 . The sector with conjugacy class $\left(\kappa^{3}\right)$ has degree shifting number 2.

The fixed point locus of $\kappa^{2}$ consists of 16 copies of $T^{2}$, given by

$$
\left\{\left(z_{1}, z_{2}, z_{3}\right)+\Lambda: z_{1} \in \mathbf{C}, z_{2}, z_{3} \in\left\{0, \frac{1}{2}, \frac{i}{2}, \frac{1}{2}+\frac{i}{2}\right\}\right\}
$$

Twelve of the 16 copies of $T^{2}$ fixed by $\kappa^{2}$ are identified in pairs by the action of $\kappa$, and these contribute 6 copies of $T^{2}$ to the singular set of $T^{6} / \mathbf{Z}_{4}$. On the remaining 4 copies $\kappa$ acts as -1 , so these contribute 4 copies of $S^{2}=T^{2} /\{ \pm 1\}$ to the singular set. The degree shifting number of these 2-dimensional twisted sectors is 1 .

Next, we construct inner local systems. We start with two-dimensional twisted sectors. Since $\kappa^{-2}=\kappa^{2}$, condition (2) of Definition 3.1 tells us that the flat orbifold line bundle $L$ over two-dimensional sectors has the property $L^{2}=1$. Now, we assign trivial line bundles to all $T^{2}$-sectors and $k(k=0,1,2,3,4) S^{2}=T^{2} /\{ \pm 1\}$-sectors. For the remaining $S^{2}=T^{2} /\{ \pm 1\}$-sectors, we assign a flat orbifold line bundle $T^{2} \times \mathbf{C} /\{ \pm 1\}$. For the zero-dimensional sectors, they are all points of two-dimensional sectors. If we assign a trivial bundle on a twodimensional sector, we just assign the trivial bundle to its point sectors. For these two- dimensional sectors with nontrivial flat line bundle, we need to be careful to choose the flat orbifold line bundle on its point sectors to ensure the condition (3) of Definition 3.1. Suppose that $\Sigma$ is one of the 2-dimensional sectors supporting nontrivial flat orbifold line bundle. It contains four singular points which generate the point sectors. Let $x$ be one of these four points. The point $x$ generates two sectors given by the conjugacy classes $(\kappa),\left(\kappa^{3}\right)$. For condition $(3)$, we have to consider the conjugacy class of the triple $\left(g_{1}, g_{2}, g_{3}\right)$ with $g_{1} g_{2} g_{3}=1$. The only nontrivial choices are $(\mathbf{g})=\left(\kappa, \kappa, \kappa^{2}\right),\left(\kappa^{2}, \kappa^{3}, \kappa^{3}\right)$. The corresponding components of $X_{(\mathrm{g})}$ are exactly these singular points. Clearly, $x$ is fixed by the whole group $\mathbf{Z}_{4}$. The orbifold line bundle is given 
by the action of $\mathbf{Z}_{4}$ on $\mathbf{C}$. Consider the component of $X_{(\mathrm{g})}$ generated by $x$. The pull-back of the flat orbifold line bundle from the 2-dimensional sector $\left(\left(\kappa^{2}\right)\right.$-sector $)$ is given by the action $\kappa v=-v$. A moment's thought tells us that for the sectors $(\kappa),\left(\kappa^{3}\right)$, we should assign a flat orbifold line bundle given by the action of $\mathbf{Z}_{4}$ on $\mathbf{C}$ as $\kappa v=i v$. It is easy to check that for the above choices the condition (3) is satisfied for $X_{(\mathrm{g})}$. It is easy to check condition (4) for our choice. Therefore, the twisted sectors given by $(x,(\kappa)),\left(x,\left(\kappa^{3}\right)\right)$ give no contribution to twisted orbifold cohomology. Suppose that the resulting local system is $\mathbf{L}_{k}$. For the sectors with trivial line bundle, they contribute $6+k$ generators to $H_{\text {orb }}^{1,1}, H_{\text {orb }}^{2,2}$ and 6 generators to $H_{\text {orb }}^{2,1}, H_{\text {orb }}^{1,2}$. Its point sectors contribute $4 k$ generators to $H_{o r b}^{1,1}, H_{\text {orb }}^{2,2}$. The remaining sectors contribute $4-k$ generators to $H_{o r b}^{2,1}, H_{o r b}^{1,2}$. Its point sectors give no contribution. Moreover, the nontwisted sector contributes

$$
h^{0,0}=h^{3,3}=2, h^{1,1}=5 .
$$

In summary, we obtain

$$
\begin{gathered}
\operatorname{dim} H_{\text {orb }}^{0,0}\left(T^{6} / Z_{4}, \mathbf{L}_{k}\right)=\operatorname{dim} H_{\text {orb }}^{3,3}\left(T^{6} / Z_{4}, \mathbf{L}_{k}\right)=1, \\
\operatorname{dim} H_{\text {orb }}^{1,1}\left(T^{6} / Z_{4}, \mathbf{L}_{k}\right)=\operatorname{dim} H_{\text {orb }}^{2,2}\left(T^{6} / Z_{4}, \mathbf{L}_{k}\right)=11+5 k \\
\operatorname{dim} H_{\text {orb }}^{1,2}\left(T^{6} / Z_{4}, \mathbf{L}_{k}\right)=\operatorname{dim} H_{\text {orb }}^{2,1}\left(T^{6} / Z_{4}, \mathbf{L}_{k}\right)=12-k
\end{gathered}
$$

Our calculation matches the Betti numbers of Joyce's desingularizations.

The orbifold cohomology rings of the following examples have been computed in $[\mathbf{C R}]$. Here, we compute their twisted version.

Example 6.4: Let's consider the case that $X$ is a point with a trivial group action of $G$. Suppose that $\alpha \in H^{2}(G, U(1))$ is a discrete torsion. We want to compute $H_{o r b, \alpha}^{*}(X, \mathbf{C})$. The twisted sector $X_{(g)}$ is a point with group $C(g)$. It is obvious that $H^{0}\left(X_{(g)}, L_{g}^{\alpha}\right)=0$ unless $L_{g}^{\alpha}=1$. Recall that a conjugacy class $(g)$ is $\alpha$-regular iff $L_{g}^{\alpha}=1$. Hence, only the $\alpha$-regular classes will contribute. Therefore, orbifold cohomology is generated by $\alpha$-regular conjugacy classes of elements of $G$, which is also the case for the center of the twisted group algebra $\mathbf{C}_{\alpha}[G]$. From Theorem 5.3, it is clear that the product is precisely the multiplication of the twisted group algebra. Therefore, as a ring $H_{o r b, \alpha}^{*}(X, \mathbf{C})$ is isomorphic to the center of the twisted group algeba $\mathbf{C}_{\alpha}[G]$. 
Example 6.5: $\quad$ Suppose that $G \subset S L(n, \mathbf{C})$ is a finite subgroup. Then, $\mathbf{C}^{n} / G$ is an orbifold. Suppose that $\alpha \in H^{2}(G, U(1))$ is a discrete torsion. For any $g \in G$, the fixed point set $X_{g}$ is a vector subspace and $X_{(g)}=X_{g} / C(g)$. By definition, $L_{(g)}=X_{g} \times \gamma(\alpha)_{g}$ C. Therefore, $H^{*}\left(X_{(g)}, L_{(g)}\right)$ is the subspace of $H^{*}\left(X_{g}, \mathbf{C}\right)$ invariant under the twisted action of $C(g)$,

$$
h \circ \beta=\gamma(\alpha)_{g}(h) h^{*} \beta
$$

for any $h \in C(g), \beta \in H^{*}\left(X_{g}, \mathbf{C}\right)$. However, $H^{i}\left(X_{g}, \mathbf{C}\right)=0$ for $i \geq 1$. Moreover, if $\gamma(\alpha)_{g}$ is nontrivial, $H^{0}\left(X_{g}, L_{(g)}\right)=0$. Therefore, $H_{o r b}^{p, q}=0$ for $p \neq q$ and $H_{o r b}^{p, p}$ is a vector space generated by the conjugacy classes of $\alpha$-regular elements $g$ with $\iota_{(g)}=p$. Therefore, we have a natural decomposition

$$
H_{o r b, \alpha}^{*}(X, \mathbf{C})=Z\left[\mathbf{C}_{\alpha}[G]\right)=\sum_{p} H_{p}
$$

where $H_{p}$ is generated by the conjugacy classes of $\alpha$-regular elements $g$ with $\iota_{(g)}=p$. The ring structure is also easy to describe. Let $x_{(g)}$ be the generator corresponding to the zero cohomology class of the twisted sector $X_{(g)}$ such that $g$ is $\alpha$-regular. The cup product is exactly same as in the nontwisted case except we replace conjugacy class by $\alpha$-conjugacy class, and multiplication of the group algebra by the multiplication of the twisted group algebra.

\section{References}

[CR] W. Chen and Y. Ruan, A new cohomology theory for orbifold, math.AG/0004129

[CR1] W. Chen and Y. Ruan Orbifold quantum cohomology, math.AG/0005198

[D] R. Dijkgraaf, Discrete torsion and symmetric product, hep-th 9912101

[JO] D. Joyce, On the topology of desingularizations of Calabi-Yau orbifolds, math.AG/9806146

[K] R. Kaufmann, Orbifolding Frobenius algebras, math.AG/0107163

[SC] P. Scott, The geometries of 3-manifolds, Bull. London. Math. Soc., 15 (1983), 401-487.

[T] W. Thurston, Three-dimensional geometry and topology, Princeton Lecture Notes

[VW] C. Vafa and E. Witten, On orbifolds with discrete torsion, J.Geom.Phys. $15(1995) 189$ 
Department of Mathematics, University of Wisconsin-Madison MADISON, WI 53706

Partially supported by the National Science Foundation . 